\title{
The Significance of Urinary RBP and KIM-1 Determination in Cisplatin - Induced Acute Kidney Injury
}

\author{
Yang Haoqiang, Meng Xiaoyan, Huang Xiangyang, Tan Hechang, Zhang Min, Shu Tingting* \\ Department of Nephrology, Liuzhou Worker's Hospital, Liuzhou City, China \\ Email address: \\ 93029876@qq.com (Shu Tingting) \\ ${ }^{*}$ Corresponding author \\ To cite this article: \\ Yang Haoqiang, Meng Xiaoyan, Huang Xiangyang, Tan Hechang, Zhang Min, Shu Tingting. The Significance of Urinary RBP and KIM-1 \\ Determination in Cisplatin - Induced Acute Kidney Injury. Science Journal of Clinical Medicine. Vol. 10, No. 2, 2021, pp. 47-51. \\ doi: $10.11648 /$ j.sjcm.20211002.16
}

Received: May 6, 2021; Accepted: May 24, 2021; Published: June 25, 2021

\begin{abstract}
Objective: Cisplatin is one of the most commonly used and most effective chemotherapy drugs in clinical treatment of solid tumors, because of its strong anti-cancer effect, no cross resistance with other cancer drugs, but cisplatin is one of the significant renal toxicity drugs either, which greatly limits its clinical application. The traditional diagnostic methods for AKI are to measure Scr and changes in urine volume, but these indicators are affected by non-renal factors and cannot reflect renal function in an early and timely manner, which delays the optimal early treatment time for AKI. To discuss the clinical value of detecting urinary retinol binding protein (RBP) and kidney injury molecule 1 (KIM-1) in early diagonsis of cisplatin-induced acute kidney injury. Methods: 48 rats were randomly divided into the cisplatin group (CP group, 42 rats) and the control group (NS group, 6 rats). The urinary RBP and KIM-1, renal pathological changes were observed at each injection time points. Results: Urinary KIM-1 it significantly increased at $12 \mathrm{~h}$ point $(\mathrm{P}<0.05)$, which was higher obviously than NS group. Urinary RBP it significantly elevated at $6 \mathrm{~h}$ point $(\mathrm{P}<0.05)$. It has correlation with Scr. Conclusion: In cisplatin-induced acute kidney injury, urinary RBP and KIM-1 increased obviously earlier than serum creatinine, they were early sensitive markers.
\end{abstract}

Keywords: Cisplatin, Acute Kidney Injury, Urinary Retinol Binding Protein, Kidney Injury Molecule 1

\section{Introduction}

With the rapid development of the pharmaceutical industry in recent years, new drugs have been applied in clinical practice, and drug-induced AKI has been increasing year by year, which has become an unavoidable problem for clinical medicine workers. Cisplatin is one of the most commonly used and most effective chemotherapy drugs in clinical treatment of solid tumors, because of its strong anti-cancer effect, no cross resistance with other cancer drugs, as well as the most commonly used antitumor drug combination chemotherapy. Its antitumor effect is positively correlated with dose, but cisplatin is one of the significant renal toxicity drugs either, and renal toxicity was positively correlated with its dosage. In other words, the higher the dose of cisplatin, the better the anti-tumor effect, at the same time, the stronger the nephrotoxicity, the greater the probability of renal injury. Clinical investigation shows that the incidence of cisplatin renal damage as much as $25 \%-35 \%$ [1-5]. Therefore, its clinical application is greatly limited. How to effectively ensure the quality of chemotherapy for cancer patients, reduce kidney injury, and better play the anti-tumor effect of cisplatin has become an urgent problem to be solved. The traditional diagnosis of AKI is mainly based on SCR or changes in urine volume, but there are many factors affecting these indicators [6]. Studies have shown that SCR increases only when glomerular filtration rate drops by more than $50 \%$, which cannot achieve the purpose of early diagnosis and delays the optimal treatment time for AKI. Therefore, it is an urgent requirement for new drug development and clinical treatment to find early, sensitive and quantifiable AKI markers.

\section{Materials and Methods}

\subsection{Animals}

Healthy adult male SD rats, weighing 200-220g, SPF grade, were purchased from Experimental Animal Center of Guangxi 
Medical University. Rats were kept in the Animal Experiment Center of the Fourth Affiliated Hospital of Guangxi Medical University. The temperature of the animal center was set at $20^{\circ} \mathrm{C}-28^{\circ} \mathrm{C}$, and the air humidity was controlled at $50 \%-60 \%$. The light and darkness were alternated for $12 \mathrm{~h}$, and the rats were separately caged and fed freely within $24 \mathrm{~h}$. A total of 48 adult SD rats were included, including 42 in $\mathrm{CP}$ group and 6 in NS group.

\subsection{Treatment}

The experiment was divided into CP group and NS group. The CP group was divided into CP3h group, CP6h group, $\mathrm{CP} 12 \mathrm{~h}$ group, $\mathrm{CP} 24 \mathrm{~h}$ group, $\mathrm{CP} 48 \mathrm{~h}$ group, $\mathrm{CP} 72 \mathrm{~h}$ group and CP96h group according to different time after injection, with 6 rats in each group; After adaptive feeding for 3 days, SD rats were divided into 8 groups according to random number method, and randomly assigned to CP group and NS group.

\subsection{Material}

$6 \mathrm{ml} / 30 \mathrm{mg}$ cisplatin injection from Jiangsu Hausen Pharmaceutical Co., Ltd. Batch number: 120318.

\subsection{Definition and Diagnostic Criteria of AKI}

\subsubsection{Definition of AKI}

The Acute Kidney Injury Network (AKIN) Panel defines AKI as abnormalities in renal function or structure not exceeding 3 months, including abnormalities in blood, urine, and tissue testing or imaging markers of renal injury.

\subsubsection{Diagnostic Criteria for AKI}

The AKIN consensus determined the diagnostic criteria for AKI as follows: sudden renal function decrease within 48 hours, and Scr increase with absolute value $>26.5 \mu \mathrm{mol} / \mathrm{L}(0.3 \mathrm{mg} / \mathrm{dL})$; Or Scr increased by $>50 \%$ compared with the original level; And/or decreased urine volume $<0.5 \mathrm{ml} /(\mathrm{kg} \bullet \mathrm{h})$ for more than 6 hours, obstructive nephropathy or dehydration were excluded [4].

\subsection{Methods}

1) The rat model of cisplatin AKI was established by single intraperitoneal injection of $10 \mathrm{mg} / \mathrm{Kg}$ cisplatin to SD rats in CP group [15], NS group SD rats were intraperitoneally injected with $2 \mathrm{ml} / \mathrm{Kg}$ normal saline as control group.

2) At each time point after drug injection, SD rats were subjected to ventral median incision after ether inhalation anesthesia, and $5 \mathrm{ml}$ of blood was collected by portal venipuncture and put into EP tube and sent to the laboratory of our hospital for Scr and BUN determination; $0.8-1 \mathrm{ml}$ of urine was taken by bladder puncture and sent to the laboratory of our hospital for urine RBP and KIM-1 determination; bilateral kidneys were taken and sent to the pathology department of our hospital for HE pathological section.

\subsection{Investigated Rats Parameters}

1) General situation of rats: the body weight of rats in $C P$ group and NS group, death during the experiment, mental condition, hair condition, activity and feeding condition of rats in each group and their changes over time were observed;

2) Blood and urine biochemical indexes: The detection values of Scr, BUN, urinary RBP, and urinary KIM-1 were observed at each time point, and the change trend of Scr, urinary RBP, KIM-1 and whether there was correlation between urinary RBP, KIM-1 and Scr;

3) HE staining of renal tissue pathology: observe the structure of glomerulus and renal tubule in renal $\mathrm{HE}$ section, especially observe whether the epithelial cells of renal tubule shed like brush edge, cytoplasmic vacuolar degeneration, lumen dilation, renal tubule blockage, interstitial inflammatory cell infiltration and apoptotic cells.

\subsection{Statistical}

All measurement data were expressed as mean plus or minus standard deviation ( $x_{ \pm \mathrm{S}}$ ), and analyzed by SPSS17.0 statistical software. Block design analysis of variance was used for multivariate comparison of group design, LSD test or SNK test was used for pairwise comparison of multiple mean within the group, and Spearman correlation analysis was used for correlation analysis of measurement data. The test level was set as 0.05 , and $\mathrm{P}<0.05$ was statistically significant.

Table 1. Scr, BUN, KIM-1 and RBP.

\begin{tabular}{|c|c|c|c|c|c|c|}
\hline Group & & $\mathbf{N}$ & $\operatorname{Scr}(\mathrm{mol} / \mathrm{l})$ & BUN (mmol/l) & KIM-1 (ng/ml) & RBP (mg/l) \\
\hline NS & & 6 & $45.733 \pm 4.541$ & $5.129 \pm 0.913$ & $1.375 \pm 0.432$ & $4.367 \pm 0.659$ \\
\hline \multirow{6}{*}{$\mathrm{CP}$} & $3 \mathrm{~h}$ & 6 & $45.133 \pm 4.301$ & $5.076 \pm 1.075$ & $1.361 \pm 0.408$ & $4.467 \pm 0.596$ \\
\hline & $6 \mathrm{~h}$ & 6 & $45.083 \pm 4.354$ & $5.147 \pm 1.149$ & $1.933 \pm 0.501$ & $6.050 \pm 0.909^{\star}$ \\
\hline & $12 \mathrm{~h}$ & 6 & $45.400 \pm 4.392$ & $5.139 \pm 1.342$ & $2.769 \pm 0.532^{\star}$ & $7.317 \pm 1.408^{\star}$ \\
\hline & $24 \mathrm{~h}$ & 6 & $47.400 \pm 5.653$ & $5.253 \pm 1.491$ & $3.633 \pm 0.585^{\star}$ & $8.800 \pm 1.820^{\star}$ \\
\hline & $48 \mathrm{~h}$ & 6 & $59.083 \pm 6.956^{\star \nabla}$ & $6.644 \pm 1.647^{\star \nabla}$ & $6.667 \pm 1.256^{\star \nabla}$ & $10.783 \pm 1.992^{\star}$ \\
\hline & $96 \mathrm{~h}$ & 6 & $96.950 \pm 11.265^{\star \nabla}$ & $10.217 \pm 2.638^{\star \nabla}$ & $2.183 \pm 0.560^{\star}$ & $12.917 \pm 2.395^{\star}$ \\
\hline$F$ values & & & 56.353 & 21.647 & 20.726 & 45.693 \\
\hline$P$ values & & & 0.000 & 0.000 & 0.000 & 0.000 \\
\hline
\end{tabular}

$\star$ comparison between CP group and NS $\mathrm{P}<0.05$,

$\checkmark$ comparison within $\mathrm{CP}$ group, $\mathrm{P}<0.05$. 


\section{Results}

\subsection{General Situation of Rats}

After injection, the body weight of rats in CP group and NS group showed no significant change, and no death occurred. However, SD rats in CP group showed listlessness, sparse yellow and dull hair color, lazy eye closure, reduced food intake and other phenomena, and the above phenomena increased with time.

\subsection{Morphological Structure}

HE staining of kidney tissue in NS group showed clear renal tissue structure without obvious abnormality, normal glomeruli and renal tubule structure with less interstitium; No obvious abnormalities were observed in glomeruli and renal tubules in the $\mathrm{CP} 3 \mathrm{H}, \mathrm{CP} 6 \mathrm{H}$ and $12 \mathrm{H}$ groups. In $\mathrm{CP} 24 \mathrm{~h}$ group, no obvious glomeruli abnormality was observed, but some renal tubules showed vacuolar degeneration. No obvious glomeruli abnormality was observed in the CP48h group, but tubular vacuolar degeneration was significantly worse than that in the $\mathrm{CP} 24 \mathrm{~h}$ group. There was no obvious glomeruli abnormality in the $\mathrm{CP} 72 \mathrm{~h}$ group, but the renal tubular degeneration was worse than that in the $\mathrm{CP} 48 \mathrm{~h}$ group, and inflammatory cell infiltration was observed in the renal interstitium. In the CP96h group, there was erythrocyte stasis in the glomeruli, but no obvious abnormity was observed. However, the epithelial cells of the renal tubules showed obvious brush edge abscission, and the vacuolar degeneration of the cytoplasm was significantly worse than before. In addition, the lumen dilated significantly, with the appearance of tubules and even the aggregation of apoptotic cells, as shown in Figure 1.

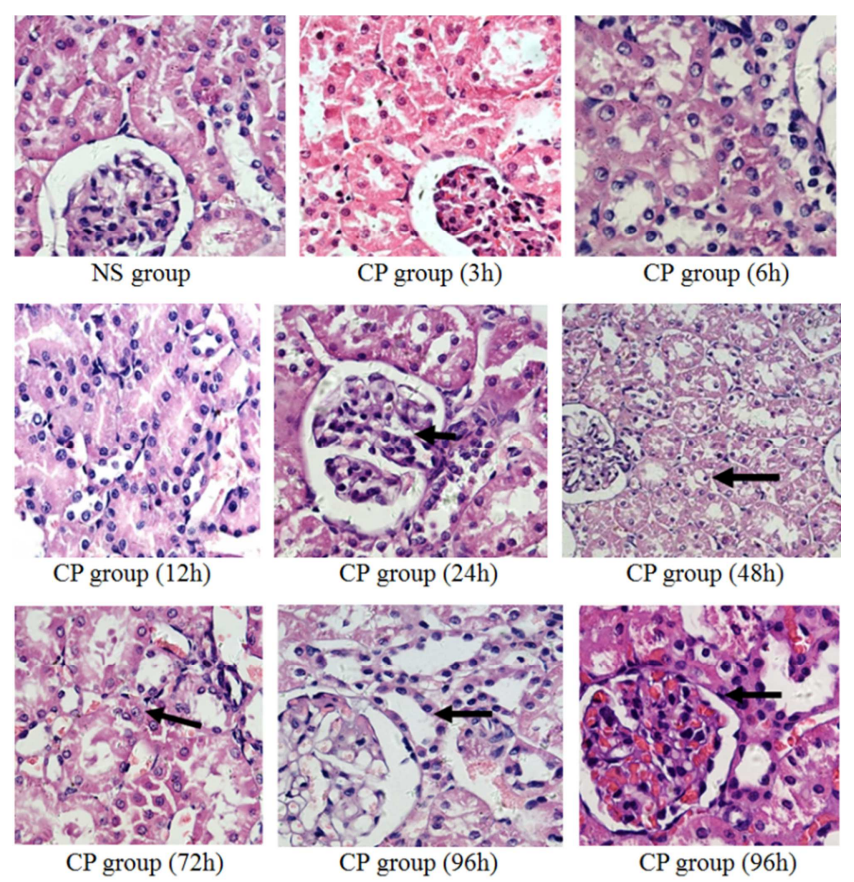

Figure 1. HE staining Results $(H E \times 400)$.

\section{Discussion}

Cisplatin is a widely used chemotherapeutic agent for periodic nonspecific heavy metal tumors, and it is also the most commonly used anti-tumor drug in combination chemotherapy because it has no cross resistance with other tumor drugs [7-8]. Cisplatin has high accumulation, high metabolism and high excretion in the kidney, and its nephrotoxicity is particularly prominent, which is positively correlated with the drug dosage. Clinical statistics show that the incidence of AKI during cisplatin chemotherapy is as high as $35 \%$, which greatly limits its clinical application. The traditional diagnostic methods for AKI are to measure Scr and changes in urine volume, but these indicators are affected by non-renal factors and cannot reflect renal function in an early and timely manner, which delays the optimal early treatment time for AKI. Therefore, it is an urgent requirement for the development of The Times to find early, sensitive and quantifiable AKI markers.

$\mathrm{CP}$ group of Scr in this study results suggest that $\mathrm{CP}$ group of SD rats abdominal cavity injection of cisplatin, within $24 \mathrm{~h}$ when Scr no obvious change, began to rise within $24 \mathrm{~h}-48 \mathrm{~h}$, $48 \mathrm{~h}$ was significantly higher compared with the NS control group, the difference was statistically significant. During $48 \mathrm{~h}$ (24h-72h) SCR increased $>$ by $50 \%$ relative to baseline, and no death in rats, conform to the Scr rise within $48 \mathrm{~h}>50 \%$ AKI diagnosis standard, indicates that under the condition of this experiment, cisplatin, $10 \mathrm{mg} / \mathrm{kg}$ to create a single intraperitoneal injection drug AKI cisplatin SD rats model was established, at the same time $72 \mathrm{~h}$ can be diagnosed AKI class I, AKI grade II can be diagnosed at $96 \mathrm{~h}$. The results of this study are consistent with the results of Vikash Sinha et al. that Scr of rat AKI model was significantly increased within 3 days after $5.5 \mathrm{mg} / \mathrm{Kg}$ cisplatin was injected into the rat tail vein [9-10].

The results of BUN in CP experimental group showed that no significant change of BUN was observed within $24 \mathrm{~h}$, but it began to rise within $24 \mathrm{~h}-48 \mathrm{~h}$ and was significantly higher than that in NS control group at $48 \mathrm{~h}$, and the difference was statistically significant. Scr and BUN are both evaluation indexes of glomerular filtration rate impairment of renal function. The results of this study indicated that no significant changes were observed in both of them within $24 \mathrm{~h}$, but showed a continuous upward trend within $24 \mathrm{~h}$ to $96 \mathrm{~h}$, indicating that renal function of rats in this study was continuously impaired and gradually aggravated.

Large Numbers of clinical studies and basic studies show that cisplatin acute kidney injury kidney disease mainly for renal tubular epithelial cells dysfunction, a main mechanism is to cause kidney toxicity: cisplatin can cause renal tubular epithelial cells in ischemia and directly lead to the lack of oxygen, if damage is severe and no intervention will eventually lead to renal tubular epithelial cell necrosis, which in turn causes kidney structure change; The other mechanism is to cause renal ischemia: cisplatin can cause renal vasoconstriction, resulting in decreased renal blood flow and glomerular filtration rate, thereby causing renal injury. The 
results of HE staining of kidney pathology in this experiment indicated that, compared with the NS group, no obvious abnormalities were observed in the glomeruli of the CP group except erythrocyte deposition. Renal tubular of NS group not seen obvious abnormity, and $\mathrm{CP}$ group rat renal tubule degeneration obviously. In the early stage, the main manifestation was renal tubular vacuolar degeneration, further development of renal tubular epithelial cells, loss of brush border, malpighian tube cavity expansion, interstitial inflammatory cell infiltration, in the absence of interference will gradually develop to control cavity exposed basement membrane, red and white cell or protein appeared on renal tubular plug pipe type, eventually lead to renal tubular epithelial cell apoptosis; The change trend of renal tubules in this study was consistent with the change of Scr and BUN, that is, the results of this study confirmed that the renal toxicity target of cisplatin was mainly on renal tubules rather than glomeruli. The results of this study were consistent with the results of Gravedi P, Gaspari F and Mori K, and Mishra J et al.'s study on renal tubules as the main renal lesions in acute renal injury with cisplatin [11-12].

$\mathrm{RBP}$ is a low molecular weight lipophile carrier protein synthesized and secreted by the rough endoplasmic reticulum of the liver, with a molecular weight of $21 \mathrm{KD}$, a $\mathrm{pH}$ of $4.4-4.8$ and a half-life of $3-12 \mathrm{~h}$. The main function of RBP is to transport retinol from the liver to epithelial cells, and can specifically bind to retinal epithelial cells to provide retinol to the retina. RBP is widely distributed in human serum, cerebrospinal fluid, urine and other body fluids, since it has the characteristics of small molecular weight and short half-life, in the liver, it has important clinical significance in early diagnosis and curative effect observation of kidney disease. And because RBP can reflect the body's nutritional status in specificity, it is also a diagnosis early sensitive indicator of malnutrition. In the blood, RBP and retinol, prealbumin In the form of a 1:1:1 (mol) polymer complex and transports $90 \%$ of retinol in the body, it can't be filtered by the glomerular membrane. After retinol is transported to the target cell and binds to the RBP receptor on the surface of the target cell, complex dissociation, retinol into cells, RBP was free in the plasma, was quickly glomerular filtration, almost all in renal proximal convoluted tubules heavy absorption, and broken down into amino acids for organizations to use, only a small amount is excreted in the urine. Under normal circumstances, RBP has strong stability in urine, is not easily decomposed, is not affected by $\mathrm{pH}$ and blood pressure, and the excretion of RBP is very little $<0.7 \mathrm{mg} / \mathrm{L}$. When the function of the near convolved tubule is impaired, its reabsorption function is decreased, and the urinary RBP can be increased, so it is also recognized as one of the early biomarkers of AKI. Due to the numerous etiology of AKI, the types and changes of biological markers of AKI caused by different etiology are different. In AKI caused by nephrotoxic drug cisplatin, no urine RBP has been reported.

When the kidney is damaged by cisplatin, renal tubule function is impaired, leading to RBP reabsorption disorder, which leads to the increase of urinary RBP, and the more severe the renal tubule injury is, the higher the urinary RBP is. In this study, the RBP results in the CP group indicated that there was no obvious abnormality in urine RBP within $3 \mathrm{~h}$, but it began to rise within $3 \mathrm{~h}-6 \mathrm{~h}$, and the difference at $6 \mathrm{~h}$ was statistically significant compared with that in the NS group, showing a continuous upward trend within 6h-96h. In this study, the results of RBP in CP group indicated that the abnormal increase of RBP could be detected after $6 \mathrm{~h}$, which was significantly earlier than the increase of SCR, indicating that urinary RBP was as sensitive as KIM-1 in cisplatin acute renal injury as SCR. The results of this study are consistent with Zhong Fangcai et al. 's study that urinary RBP is a sensitive marker for acute kidney injury and Wang Yongqing et al.' s study that urinary RBP is a sensitive marker for acute kidney injury in tumor chemotherapy.

According to RBP and Scr related statistical analysis indicate that the urine RBP and Scr were positively correlated, the correlation coefficient of $\mathrm{r}=0.964(\mathrm{P}=0.000<0.05)$, indicated that the higher the degree of renal injury, the higher the SCR and the more serious the renal tubule lesion, the higher the urine RBP. It means the urine RBP can reflect cisplatin in rats with acute kidney injury degree of kidney damage, consistent with the experimental Scr and pathology results, and Huang Yunfang wen-li Chen et al. Study of RBP in AKI significance in early diagnosis and classification research results: the urine RBP can be used as evaluation index AKI illness severity.

KIM-1 is a type I transmembrane protein found in proximal renal tubular epithelial cells, belonging to the immunoglobulin gene superfamily. It is not expressed in fetal liver and kidney, slightly expressed in adult liver, kidney and spleen, but significantly enhanced expressed in kidney after ischemia injury. It is the adhesion molecule of damaged proximal confluent tubular epithelial cells, and may be involved in the process of kidney injury and repair, the development and metastasis of renal tumor, and immune response. Vaidya et al. used rat renal ischemia/reperfusion model to study and showed that KIM-1 can be used as a sensitive, specific and quantitative biological marker for early detection of renal injury [13-15].

The monitoring results of urine KIM-1 in $\mathrm{CP}$ group in this experiment showed that there was no obvious abnormality in the urine KIM-1 in CP group within 6h, but it began to rise within $6 \mathrm{~h}$ to $12 \mathrm{~h}$, and then increased significantly at $12 \mathrm{~h}$, which was significantly higher than that in NS control group. The difference was statistically significant, reaching a peak value within $24 \mathrm{~h}$ to $72 \mathrm{~h}$ and presenting a decreasing trend within $72 \mathrm{~h}$ to $96 \mathrm{~h}$. These results indicated that in the AKI model of rats induced by cisplatin, the expression of KIM-1 was significantly up-regulated within $12 \mathrm{~h}$, indicating that the increase of KIM-1 was much earlier than that of SCR, that is, KIM-1 compared with SCR was an early sensitive marker of acute kidney injury induced by cisplatin.

\section{Conclusion}

In conclusion: Renal tubule is the main target site of acute renal injury induced by cisplatin in rats, abnormal urine RBP, 
KIM - 1 rise significantly higher before the Scr, urine RBP and KIM - 1 are early sensitive markers of acute kidney injury induced by cisplatin in rats, and the urine RBP level changes can also reflect the cisplatin in rats with acute kidney injury degree of kidney damage. However, the urinary RBP and KIM-1 data obtained in this study were not corrected by urinary creatinine, and there may be deviation in the data. In addition, the interval of grouping time points was long and the total recording time was short, so the changes of SCR, urinary RBP and KIM-1 could not be recorded in detail. In future studies, we will gradually improve the experimental design, increase the number of rats and the consistency of grouping time points, make detailed records of urinary RBP and KIM-1, increase the diagnostic specificity and sensitivity and the correlation with other biomarkers, so as to obtain a more comprehensive and in-depth study of acute renal injury with cisplatin.

\section{References}

[1] Meng Xiaoyan, et al. Relationship between Apoptosis of Renal Tubular Epithelial Cells and NGAL in the Rodent Model of Cisplatin Induced Acute Kidney Injury [J]. Medical Innovation of China. 2017, 14 (05): 049-052.

[2] YangL XingG, Wang L, et al. Acute kidney injury in China: across-sectionalsurvey [J]. Lancet, 2017, 386 (10002): 1465-1471.

[3] Liu Xuewu, et al. Protective effect and mechanism of Jin shui bao tablet on acute kidney injury induced by cisplatin in rats [J]. China Pharm Univ, 2020, 51 (1): 76-83.

[4] Pang suo. et al. Advances in research of cisplatin-induced acute kidney injury. Division of Nephrology. 2020, 29 (4): 363-368.

[5] Miller pp. et al. Mechanisms of Cisplatin nephrotoxicity. Toxins (Basel), 2021, 2 (11): 2490-2518.
[6] Xi Jiaxi, Liu Xiaoxia, et al. Effects of Xueshuantong on renal function and oxidative indices in rats with cisplatin renal injury. Chinese Journal of Experimental Formulas. 2018, 18 (10): 263-266.

[7] Ramesh G, Reeves WB. Salicylate is where cisplatin nephrotoxicity by inhibition of tumor necrosis factor. Kindney Int. 2016, 65 (2): 480-498.

[8] Sun Meijuan, et al. Clinical study on the Prevention and Treatment of Renal Injury. The First Affiliated Hospital of Soochow University. 2021, 37 (1): 21-24.

[9] DeVarajan P. Nutrophil Gelatinase-Associted Llipocalin: A novel marker of kidney disease [J]. Scand J Clin Lab Invest Suppl, 2008, 2 (41): 89-94.

[10] Mehta RL, Kellum JA, Shah SV, et al. Acute Kidney Injury Network: report of an initiative to improve outcomes in Acute Kidney Injury. Ct Care, 2007, 1 (1): 31-33.

[11] Zhang L H, Zhang L H, Geng B Q, et al. Amifostine pregreatment for the protection of cisplatin-induced neprotoxicity [J]. Chin JMod Appl Pharmacy, 2008, 25 (5): 382-386.

[12] Nephrotoxicity, 2004, 23 (3): 307-315. WANG Y, WANG Y, WANG Y, et al. Aquatic hydrophoreactivity of Neutrophil gelatinase-associated lipocalin in water $[\mathrm{J}]$.

[13] Gaspari F, Gravedi P, Mandala M, et al. Predicting cisplatin-induced acute kidney by urinary neutrophil gelatinase-associated lipocalin excretion: a pilot prospective case-control study [J]. Nephron ClinPract, 2016115 (2): 154-160.

[14] Was6N E, Isoaho R, Mattila K, et al. The role of Serum cystatin $\mathrm{C}$ in the relationship to health status [J]. American Journal of Kidney Diseases, 2013, 42 (1): 36-43.

[15] Tang Hua, Zou Ping. Research progress of renal injury molecule-1 [J]. International Journal of Laboratory Medicine, 2016, 8 (27): 719-723. 